\title{
Spontaneous pneumothorax in a 24-year-old female
}

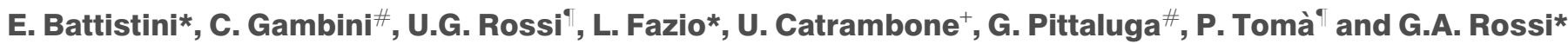

\section{CASE HISTORY}

A 24-yr-old Caucasian female was evaluated in May, 2003 at the San Martino hospital, Genoa, Italy, for the presence of "sudden onset" chest pain and nonproductive cough. Past medical history was only characterised by the presence of frequent headaches. The patient had been playing competitive tennis from the age of 6-20 yrs and, after retirement from competitions, had been smoking cigarettes $\left(0.5\right.$ pack $\cdot$ day $\left.^{-1}\right)$ for the last 4 yrs. The patient was nulliparous and reported the use of oral contraceptive in the last 3 yrs. Any suggestion of respiratory symptoms, including cough, shortness of breath or physical limitations during exercise were denied.

On admission, the patient had no dyspnoea, and physical examination demonstrated a slight decrease in breath sounds over the left hemithorax. Heart sounds were normal and there was no cyanosis, clubbing or oedema. The abdomen was nondistended, nontender, and without bruits, hepatosplenomagaly or masses. There were no focal neurological findings. The patient had normal body temperature with normal values of blood pressure, pulse and respirations and transcutaneous blood gas determination in room air showed normal arterial oxygen and carbon dioxide tension.
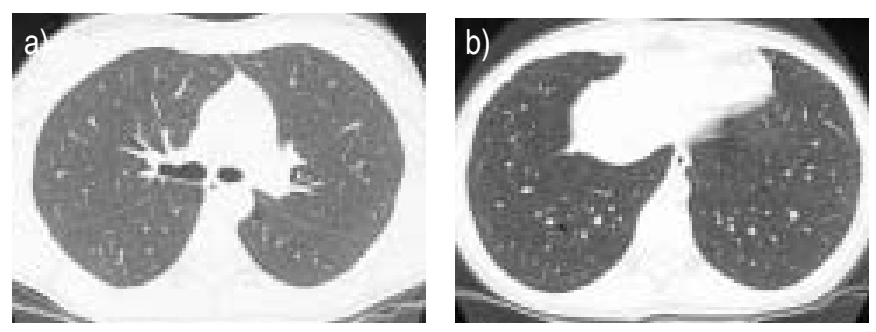

FIGURE 1. High-resolution computed tomography (CT) scans of the chest at two different levels of thoracic CT cuts performed on first admission to the outpatient clinic.
A chest radiograph demonstrated the presence of a left-sided pneumothorax. Blood tests were within normal values. After successful chest drainage, the patient was discharged.

After a period of 2 weeks, in June 2003, the patient was evaluated in the outpatient clinic at the G. Gaslini Institute, Genoa, Italy, complaining of a persistent mild pain on the left hemithorax. High-resolution computed tomography (HRCT) scans (figs $1 \mathrm{a}$ and $\mathrm{b}$ ) and pulmonary function tests (fig. 3; table 1) were performed and a follow-up re-evaluation was planned after 6-7 months. The patient did not complain of any symptoms until mid-December 2003, when a sudden onset leftsided chest pain was again experienced along with mild dyspnoea, but a chest radiograph failed to demonstrate the presence of a pneumothorax or any detectable lung abnormality. Both the chest pain and mild dyspnoea resolved without any treatment in $24 \mathrm{~h}$. As previously planned in June 2003, chest HRCT scans (figs $2 \mathrm{a}$ and $\mathrm{b}$ ) and pulmonary function tests (fig. 4; table 2) were again obtained in January, 2004 at the G. Gaslini Institute. A lung biopsy was performed and the surgical specimens were sent for morphological evaluation (fig. $5 \mathrm{a}-\mathrm{d}$ ).
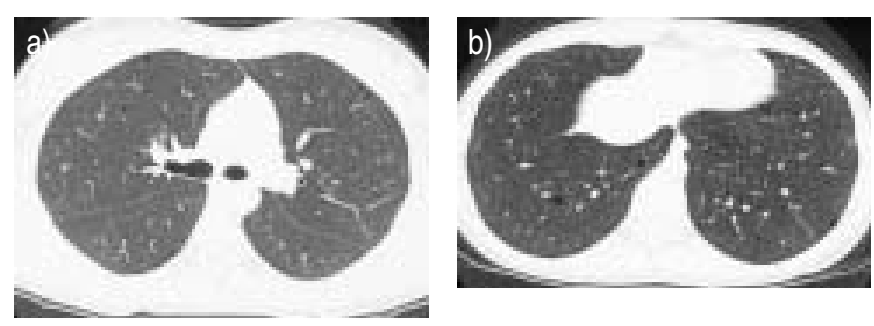

FIGURE 2. High-resolution computed tomography (CT) scans of the chest at two different levels of thoracic $\mathrm{CT}$ cuts performed on the second admission to the outpatient clinic, 7 months later.

*Pulmonary, \#Pathology, and "Radiology Units, G. Gaslini Institute, and +Thoracic Surgery Unit, San Martino Hospital, Genoa, Italy. 


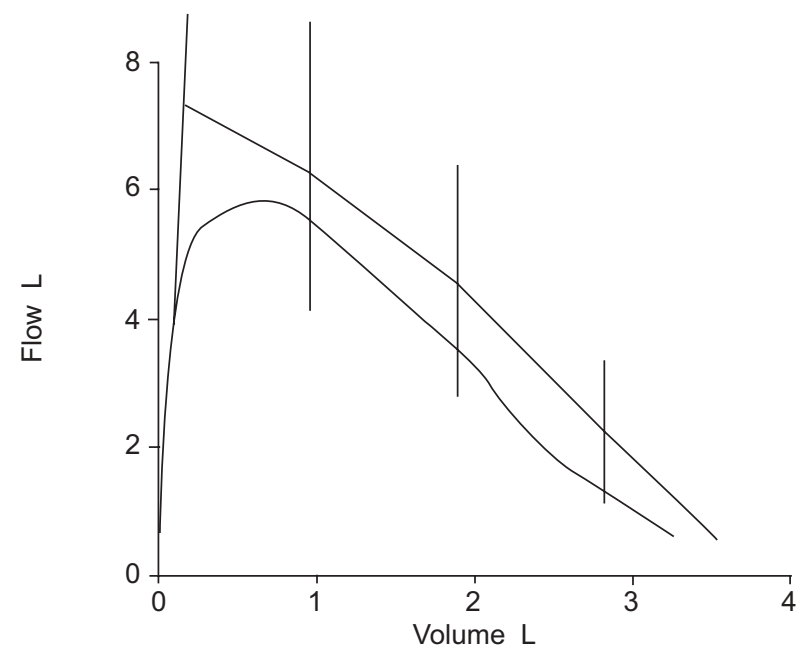

FIGURE 3. Flow-volume curve on first admission to the outpatient clinic.

\begin{tabular}{|c|c|c|c|}
\hline & Ref & Pre-Meas & Pre-\% Ref \\
\hline FVC L & 3.77 & 3.61 & 96 \\
\hline $\mathrm{FEV}_{1} \mathrm{~L}$ & 3.29 & 3.04 & 92 \\
\hline FEV $_{1 / \text { FVC } \%}$ & 84 & 84 & \\
\hline FEF $25-75 \% L \cdot \mathbf{s}^{-1}$ & 4.14 & 3.17 & 76 \\
\hline PEF L. $\mathbf{s}^{-1}$ & 7.22 & 5.81 & 80 \\
\hline
\end{tabular}

Ref: reference value for the evaluated patient; Pre-Meas: measurement evaluated at baseline (before $\beta_{2}$-adrenergic bronchodilators); Pre-\% Ref: percentage value of the parameter evaluated at baseline (before $\beta_{2}$-adrenergic bronchodilators); FVC: forced vital capacity; FEV1: forced expiratory volume in one second; FEF25-75: forced mid-expiratory flow; PEF: peak expiratory flow.

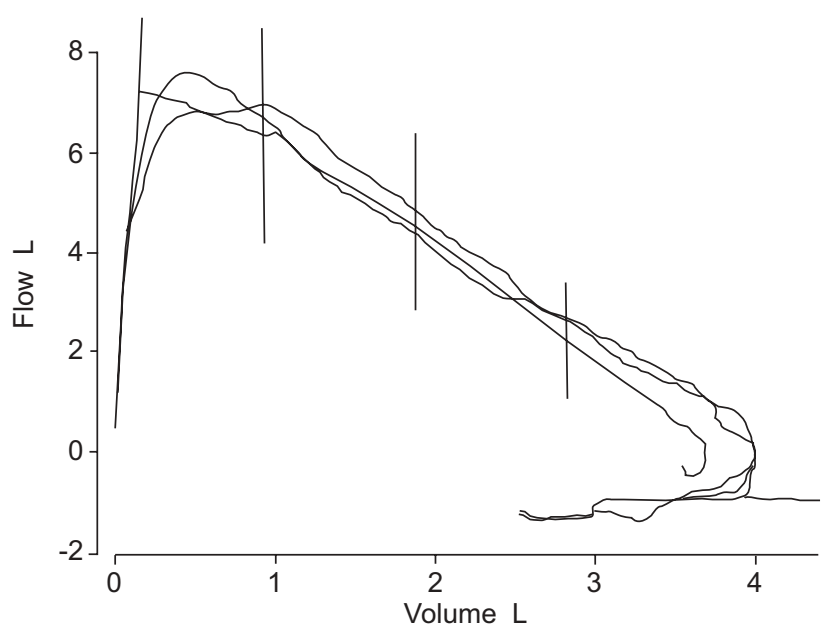

FIGURE 4. Flow-volume curve on the second admission to the outpatient clinic, 7 months later.

TABLE 2 Flow-volume curves performed on the second admission, 7 months later

\begin{tabular}{|c|c|c|c|c|c|c|}
\hline & Ref & $\begin{array}{l}\text { Pre- } \\
\text { Meas }\end{array}$ & $\begin{array}{c}\text { Pre-\% } \\
\text { Ref }\end{array}$ & $\begin{array}{l}\text { Post- } \\
\text { Meas }\end{array}$ & $\begin{array}{c}\text { Post-\% } \\
\text { Ref }\end{array}$ & $\begin{array}{c}\text { Post-\% } \\
\text { Chg }\end{array}$ \\
\hline FVC L & 3.77 & 3.99 & 106 & 3.96 & 105 & -1 \\
\hline FEV 1 L & 3.29 & 3.55 & 108 & 3.65 & 111 & 3 \\
\hline $\mathrm{FEV}_{1} / \mathrm{FVC} \%$ & 84 & 89 & & 92 & & \\
\hline FEF $25-75 \% L \cdot \mathbf{s}^{-1}$ & 4.14 & 3.85 & 93 & 4.28 & 103 & 11 \\
\hline PEF L. $\mathbf{s}^{-1}$ & 7.22 & 7.54 & 104 & 6.89 & 95 & -9 \\
\hline$D \mathrm{~L}, \mathrm{CO} \mathrm{SB} \mathrm{mL} \cdot \mathrm{min} \cdot \mathrm{mmHg}^{-1}$ & 23.58 & 21.45 & 91.0 & & & \\
\hline$D \mathrm{~L}, \mathrm{CO} / \mathrm{VA} \mathrm{mL} \cdot \mathrm{min} \cdot \mathrm{mmHg}^{-1}$ & 4.07 & 4.19 & 102.9 & & & \\
\hline
\end{tabular}

Ref: reference value for the evaluated patient; Pre-Meas: measurement evaluated at baseline (before $\beta_{2}$-adrenergic bronchodilators); Pre-\% Ref: percentage value of the parameter evaluated at baseline; Post-Meas: measurement evaluated after treatment with $\beta_{2}$-adrenergic bronchodilators; Post-\% Ref: percentage value of the predicted value of the parameter evaluated after treatment; Post-\% Chg: difference between the value of a parameter at baseline and the value of that parameter after treatment; FVC: forced vital capacity; FEV1: forced expiratory volume in one second; FEF25-75: forced midexpiratory flow; PEF: peak expiratory flow; $D \mathrm{~L}, \mathrm{CO}$ : carbon monoxide diffusing capacity of the lung; SB: single breath; VA: alveolar volume. 


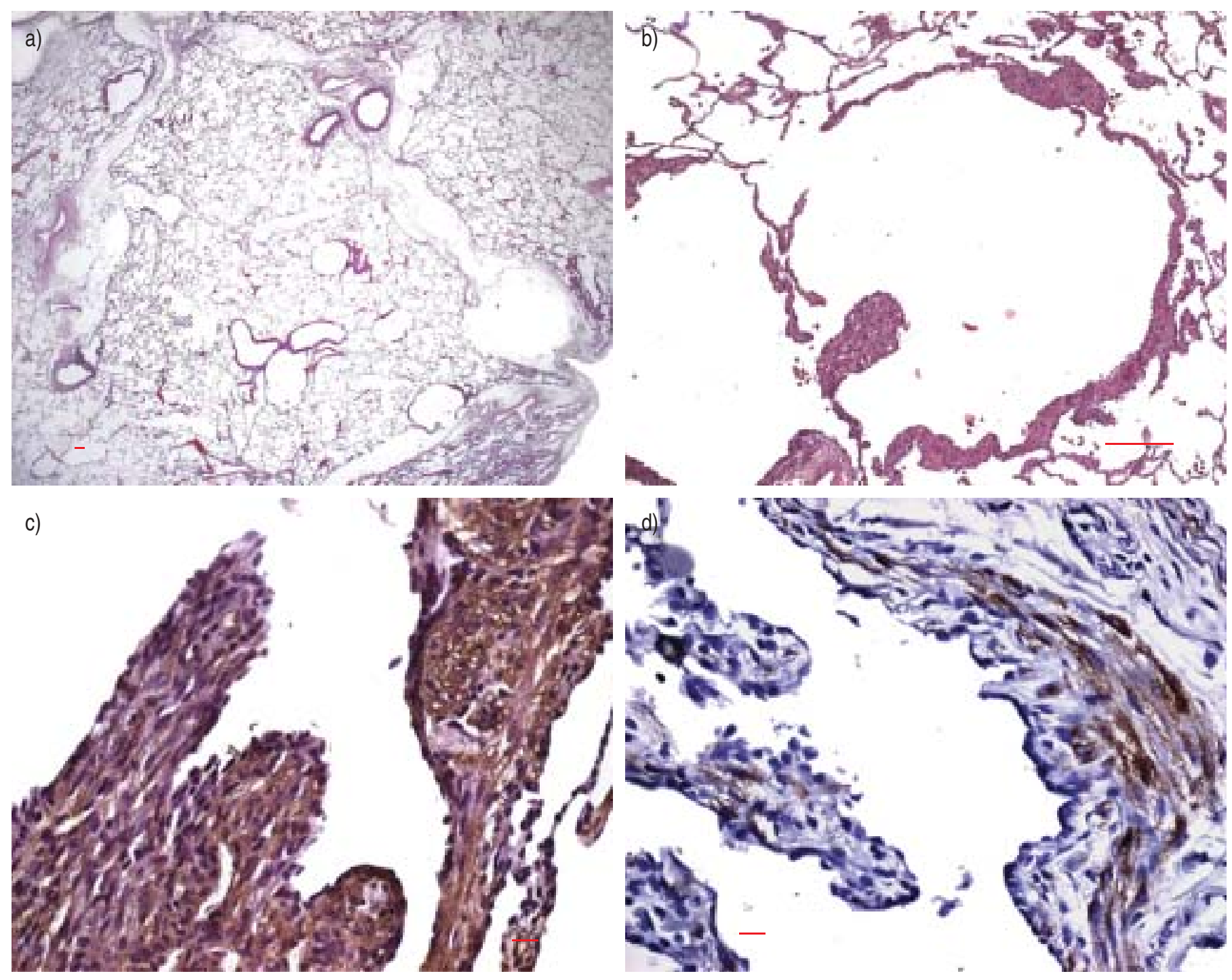

FIGURE 5. Histological preparations of lung biopsy specimen: a) haematoxylin and eosin staining at lower magnification (scale bar=100 $\mu \mathrm{m})$; b) haematoxylin and eosin staining at higher magnification (scale bar $=100 \mu \mathrm{m})$; c) immunostaining with monoclonal antibodies reacting against anti-smooth muscle action $(\mathrm{scale}$ bar $=10 \mu \mathrm{m}$ ) and d) immunostaining with the anti-melanoma-associated antigen (HMB-45) monoclonal antibody (Mab; scale bar=10 $\mu \mathrm{m}$ ).

\section{BEFORE TURNING THE PAGE, INTERPRET THE PULMONARY FUNCTION TEST DATA, COMPUTER TOMOGRAPHY SCANS AND LUNG BIOPSY RESULTS AND SUGGEST A DIAGNOSIS.}




\section{INTERPRETATION \\ HRCT and pulmonary function tests on first admission to the outpatient clinic}

Figures 1a and b show bilateral small cystic lesions of varying size, although regularly shaped, that are diffusely distributed throughout the lungs. The cystic walls are faintly perceptible. No evidence of interstitial involvement, hilar lymph node enlargement and pleural thickening or effusions was present.

Figure 3 shows the flow-volume curve, which demonstrates normal lung volumes, including forced vital capacity (FVC) and forced expiratory volume in one second (FEV1), and a slight decrease in forced mid-expiratory flow (FEF25-75\%).

\section{HRCT and pulmonary function tests on the second admission to the outpatient clinic}

Figures $2 \mathrm{a}$ and $\mathrm{b}$ show HRCT scans of the chest performed 7 months later, clearly demonstrating a progression of the cystic lesions, and an increase in their number and/or size.

Figure 4 shows the flow-volume curve demonstrating normal volumes and flows, including FVC and FEV1, and a decrease in FEF25-75\%, with increased values, as compared to those obtained in June, 2003. Carbon monoxide diffusing capacity of the lung $(D \mathrm{~L}, \mathrm{CO})$ and the $D \mathrm{~L}, \mathrm{CO}$ to alveolar volume ratio $(D \mathrm{~L}, \mathrm{CO} / V \mathrm{~A})$ were also within normal values.

\section{Diagnostic considerations}

In the differential diagnosis of the present case, the authors considered the following diagnosis: a) lymphangioleiomyomatosis; b) tuberous sclerosis (TS); and c) Langerhans cell histiocytosis or eosinophilic granuloma (LCH).

\section{Surgical procedure and interpretation of surgical specimens}

A left-sided mini-thoracotomy was performed, and multiple subpleural cystic lesions were highlighted. Biopsies of the lingula were obtained and sent to the pathologist for morphological evaluation of lung tissues.

\section{Macroscopic features}

The resected lung tissue showed a variety of small cystic lesions with thickened walls in subpleural tissue.

\section{Microscopic features}

At low magnification, ill-defined cystic lesions with thickened walls were detected in the lung parenchyma (fig. 5a). At higher magnification, the cyst walls appeared to be infiltrated by proliferating smooth muscle-like cells, which are also present in the alveolar interstitium (fig. 5b). These smooth muscle-like cells stain positive with monoclonal antibodies reacting against anti-smooth muscle actin and show a benign appearance (fig. 5c). A significant proportion of the smooth muscle cells infiltrating the lung parenchyma and the cyst walls show reactivity for the HMB-45 antigens (fig. 5d).

\section{Diagnosis: lymphangioleiomyomatosis.}

\section{Clinical course}

Staging procedures, including abdomen computed tomography $(\mathrm{CT})$ scans and ultrasonography, failed to demonstrate the presence of renal masses (e.g. angiomyolipomas) or dilatations of the abdominal lymph vessels due to lymphatic obstruction (e.g. lymphangioleiomyomas) and chylous ascites.

The patient was then referred to J. Moss, at the PulmonaryCritical Care Medicine Branch, National Heart, Lung and Blood Institute, National Institutes of Health (NIH), Bethesda, MD, USA, for further evaluation and follow-up.

The patient had two admissions at the NIH, the first in April 2004, confirming the diagnosis of lymphangioleiomyomatosis (LAM), and lack of pulmonary function abnormalities, also during cardiopulmonary exercise testing. The second visit was in September 2004, when only a mild decrease in the DL,CO values ( $D \mathrm{~L}, \mathrm{CO}$ SB: from 91 to $80 \%$; DL,CO/VA: from 102 to $91 \%$ ) were detected. The patient was well and did not complain of any symptoms at the time of writing this case study.

\section{DISCUSSION}

LAM is an uncommon parenchymal lung disease characterised by progressive cystic lung lesions, lymphatic tumours, and angiomyolipomas, primarily affecting females of childbearing age [1-3]. Onset of symptoms is most common in the 4th decade of life but may occur in late teenage yrs or those aged $>60$ yrs $[1,2,4]$. Diagnosis is made between 8 months and 22 yrs after onset of symptoms [1, 2, 4]. The present patient was 24-yrs old, and the diagnosis was established $<1$ yr after the initial symptoms. Only a small proportion of LAM patients appear to be smokers (as was the patient of the present study) or ex-smokers [2-5].

The presence of proliferating immature-appearing smooth muscle cells (referred to as LAM cells) in the lungs and in the axial lymphatic in the thorax and abdomen is considered the typical pathological hallmark of LAM [5]. Overgrowth of LAM cells may induce compression of conducting airways (leading to obstruction of airflow, air trapping, alveolar disruption, and cystic changes), pulmonary venules (causing pulmonary haemorrhage and haemosiderosis) and of lymphatic vessels (producing chylothorax and chylous ascites) [1-3, 5]. As a result of these changes, the clinical manifestations of LAM include, almost always, pleuro-pulmonary symptoms, such as chylothorax, haemoptysis, nonproductive cough, slowly progressive dyspnoea and recurrent spontaneous pneumothorax [1-3]. This latter complication occurs in $\sim 40-50 \%$ of cases [15]. In the presented case, spontaneous pneumothorax occurred in a very early stage of the disease, when the subject had no respiratory symptoms, normal pulmonary function and limited cystic lung changes. In addition to airway obstruction by proliferating smooth muscle cells, other factors may induce air leaking in the pleural space in LAM patients. These include metalloproteinases expressed and released by proliferating LAM cells, which degrade the extracellular matrix components of the lung and may contribute to the development and, possibly, the rupture of the cysts $[6,7]$.

The cystic lesions are characteristically round and thin-walled, of various sizes, and symmetrically and uniformly distributed throughout the lungs. The appearance of LAM examined by HRCT is quite characteristic, but two disorders should be considered in the differential diagnosis: TS and LCH. Pulmonary involvement in TS is indistinguishable from LAM [8]. However, isolated pulmonary TS, without extrathoracic manifestations, is rare and in the vast majority of cases, other 
clinical (mental status, skin, family history) and radiology (cranial CT) features are present to help make the correct diagnosis. In contrast, in $\mathrm{LCH}$, a disorder found predominantly in smokers, there are a number of radiographic features (pulmonary nodules, the irregular shape of the cysts, and their uneven distribution, prevalent in the upper and middle lung, with little or no involvement of the lower lobes), which are not present in LAM [9].

The profusion of cysts may be quantified by CT scans and seem to correlate with impairment of clinical conditions and functional test values $[1,4,5]$. Indeed, in LAM, evaluation of pulmonary function typically demonstrates chronic, progressive airway obstruction with increased lung volume and decreased carbon dioxide diffusion [5], leading to dyspnoea, hypoxaemia and finally to respiratory failure [4]. Since the severity of lung function impairment often closely reflects the structural/functional lung abnormalities, it was not surprising to find normal function values and no exercise-limiting symptoms in a patient with early stage disease, like the patient described in the current study $[4,5]$.

In addition to the lung structures, LAM frequently involves other organs, such as the kidneys (e.g. angiomyolipomas) and retroperitoneal lymph nodes [3, 10]. No extrathoracic alterations were detectable in the presented patient.

Light microscopy examination of lung tissue in LAM is characterised by diffuse interstitial proliferation of "immature" smooth muscle cells, frequently located in the wall of enlarged air cavities and, to a larger or lesser degree, making up part of the wall [11]. The proliferating cells are morphologically heterogeneous in shape and size, have a benign appearance and react with antibodies against smooth muscle actin, desmin, and vimentin [11, 12]. However, in contrast to normal smooth muscle cells, LAM cells and smooth muscle cells of angiomyolipomas react with the anti-melanomaassociated antigen monoclonal antibody (HMB45 Mab), which recognises a $100 \mathrm{kDa}$ glycoprotein (gp100) found in human melanoma cells [11]. Immunohistochemical study with this Mab may useful in the diagnosis, especially when evaluating very early stage disease (as in the patient presented here) or a biopsy specimen containing small numbers of these cells (as in transbronchial biopsy samples) [11, 12]. LAM cells are heterogeneous with respect to their expression of melanoma antigens; those actively proliferating, which are probably more relevant to the progression of LAM, are more likely to be negative for reactivity with the HMB45 Mab [12]. Haemosiderin deposition in the lung interstitium and the presence of haemosiderin-laden alveolar macrophages are other typical findings of LAM, the degree of these abnormalities being associated with the degree of myomatosis [13]. These changes were not detectable in the lung biopsy of the patient in the current study, possibly due to the early stage of the disease.

The overall prognosis of LAM is not good, due to the lack of effective treatment, other than lung transplantation, and death usually occurs from respiratory failure $[1-5,13]$. However, the clinical course of LAM is highly variable. Indeed, in some patients pulmonary function tests show only a slow decline (primarily impaired DL,CO and FEV1) whilst in others, loss of respiratory function is rapid, and the time from the first symptoms to onset of respiratory failure and lung transplantation may only be a few years [1-5].

Numerous strategies have been described to treat LAM [1-5, 8]. As a result of evidence of clinical worsening of the disease during pregnancy and with use of exogenous oestrogens, hormone manipulation has been extensively applied [13-15]. However, careful evaluation of studies aimed to evaluate the effects of these therapeutic approaches revealed improvement in chylothorax or chylous ascites, but no changes on the progression of pulmonary involvement and no beneficial effect on the overall course of the disease $[1-5,8,15]$.

New hopes for LAM patients are based on molecular biology approaches aimed at understanding the molecular signalling pathways responsible for aberrant LAM cell growth and tissue remodelling [16]. These studies have led to the identification of promising molecular targets for therapy, which form the basis for pilot trials now underway in Europe and the USA [17, 18].

The patient presented in this case study clearly shows that early lung changes in lymphangioleiomyomatosis may not be detected by plain chest radiography. The availability of new drugs, such as rapamycyn, able to inhibit (at least in vitro) lymphangioleiomyomatosis cell proliferation [18], will give rise to questions on the opportunity to perform computed tomography scans in females of reproductive age in case of spontaneous pneumothorax, to detect early stage disease.

\section{ACKNOWLEDGEMENTS}

The authors would like thank J. Moss, the Pulmonary-Critical Care Medicine Branch, National Heart, Lung and Blood Institute, National Institutes of Health, Bethesda, MD, USA, for critically reviewing the manuscript.

\section{REFERENCES}

1 NHLBI Workshop Summary. Report of workshop on lymphangioleiomyomatosis. National Heart, Lung, and Blood Institute. Am J Respir Crit Care Med 1999; 159: 679683.

2 Urban TJ, Lazor R, Lacronique J, et al. Pulmonary lymphangioleiomyomatosis: a study of 69 patients. Medicine (Baltimore) 1999; 78: 321-337.

3 Kalassian KG, Doyle R, Kao P, Ruoss S, Raffin TA. Lymphangioleiomyomatosis: new insights. Am J Respir Crit Care Med 1997; 155: 1183-1186.

4 Taveira-DaSilva AM, Stylianou MP, Hedin CJ, et al. Maximal oxygen uptake and severity of disease in lymphangioleiomyomatosis. Am J Respir Crit Care Med 2003; 168: 1427-1431.

5 Kitaichi M, Nishimura K, Harumi I, Izumi T. Pulmonary lymphangioleiomyomatosis: a report of 46 patients including a clinicopathologic study of prognostic factors. Am J Respir Crit Care Med 1995; 151: 527-533.

6 Hayashi T, Fleming MV, Stetler-Stevenson WG, et al. Immunohistochemical study of matrix metalloproteinases (MMPs) and their tissue inhibitors (TIMPs) in pulmonary lymphangioleiomyomatosis (LAM). Hum Pathol 1997; 28 1071-1078.

7 Matsui K, Takeda K, Yu ZX, et al. Downregulation of estrogen and progesterone receptors in the abnormal 
smooth muscle cells in pulmonary lymphangioleiomyomatosis following therapy. An immunohistochemical study. Am J Respir Crit Care Med 2000; 161: 1002-1009.

8 McCormack F, Brody A, Meyer C, et al. Pulmonary cysts consistent with lymphangioleiomyomatosis are common in women with tuberous sclerosis: genetic and radiographic analysis. Chest 2002; 121: Suppl. 3, 61 S.

9 Ryu JH, Swensen SJ. Cystic and cavitary lung diseases: focal and diffuse. Mayo Clin Proc 2003; 78: 744-752.

10 Maziak DE, Kesten S, Rappaport DC, Maurer J. Extrathoracic angiomyolipomas in lymphangioleiomyomatosis. Eur Respir J 1996; 9: 402-405.

11 Kaiserling E, Krober S, Xiao JC, Schaumburg-Lever G. Angiomyolipoma of the kidney: immunoreactivity with HMB-45: light- and electronmicroscopic findings. Histopathology 1994; 25: 41-48.

12 Matsumoto Y, Horiba K, Usuki J, Chu SC, Ferrans VJ, Moss J. Markers of cell proliferation and expression of melanosomal antigen in lymphangioleiomyomatosis. Am J Respir Cell Mol Biol 1999; 21: 327-336.

13 Rossi GA, Balbi B, Oddera S, Lantero S, Ravazzoni C. Response to treatment with an analog of the luteinizing-hormone-releasing hormone in a patient with pulmonary lymphangioleiomyomatosis. Am Rev Respir Dis 1991; 143: 174-176.

14 Sullivan EJ. Lymphangioleiomyomatosis: a review. Chest 1998; 114: 1689-1703.

15 Taylor JR, Ryu J, Colby TV, Raffin TA. Lymphangioleiomyomatosis: clinical course in 32 patients. N Engl J Med 1990; 323: 1254-1260.

16 Usuki J, Horiba K, Chu SC, Moss J, Ferrans VJ. Immunohistochemical analysis of proteins of the $\mathrm{Bcl}-2$ family in pulmonary lymphangioleiomyomatosis: association of Bcl-2 expression with hormone receptor status. Arch Pathol Lab Med 1998; 122: 895-902.

17 Carsillo T, Astrinidis A, Henske EP. Mutations in the tuberous sclerosis complex gene TSC2 are a cause of sporadic pulmonary lymphangioleiomyomatosis. Proc Natl Acad Sci USA 2000; 97: 6085-6090.

18 Goncharova EA, Goncharov DA, Eszterhas A, et al. Tuberin regulates p70 S6 kinase activation and ribosomal protein S6 phosphorylation: a role for the TSC2 tumour suppressor gene in pulmonary lymphangioleiomyomatosis (LAM). J Biol Chem 2002; 277: 30958-30967. 